PREPARED FOR THE U.S. DEPARTMENT OF ENERGY, UNDER CONTRACT DE-AC02-76CH03073

PPPL-3855

PPPL-3855

UC-70

Destabilization of Fast Magnetoacoustic Waves by Circulating Energetic lons in Toroidal Plasmas

by

V.S. Belikov, Ya.I. Kolesnichenko, and R.B. White

August 2003

M

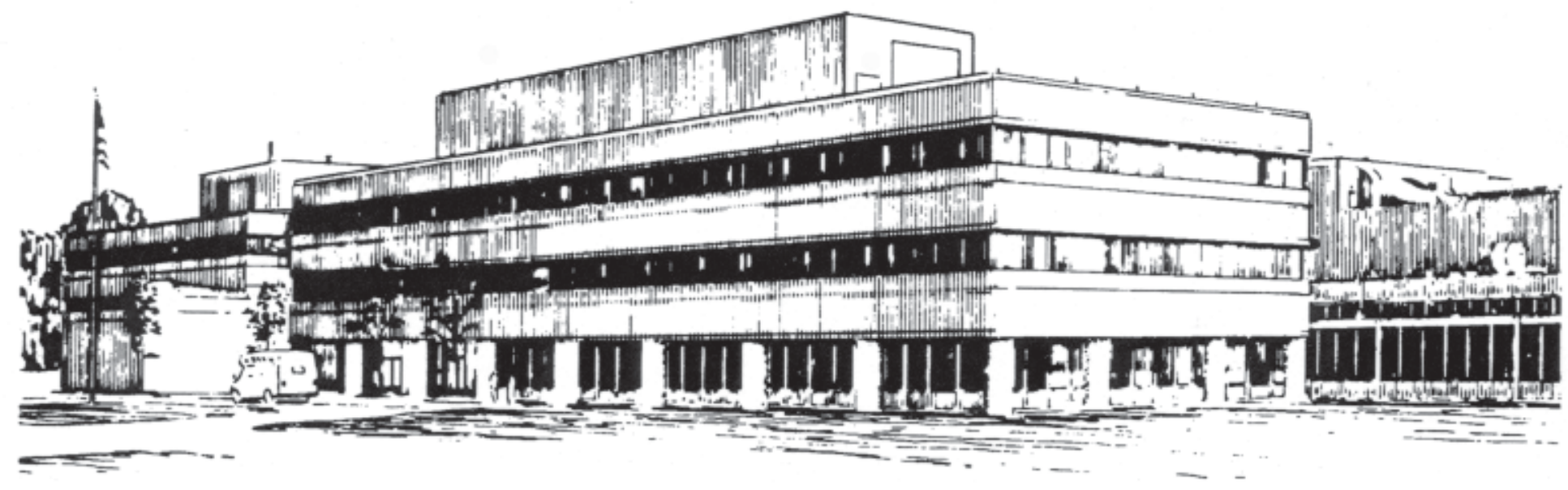

PRINCETON PLASMA PHYSICS LABORATORY PRINCETON UNIVERSITY, PRINCETON, NEW JERSEY 


\section{PPPL Reports Disclaimer}

This report was prepared as an account of work sponsored by an agency of the United States Government. Neither the United States Government nor any agency thereof, nor any of their employees, makes any warranty, express or implied, or assumes any legal liability or responsibility for the accuracy, completeness, or usefulness of any information, apparatus, product, or process disclosed, or represents that its use would not infringe privately owned rights. Reference herein to any specific commercial product, process, or service by trade name, trademark, manufacturer, or otherwise, does not necessarily constitute or imply its endorsement, recommendation, or favoring by the United States Government or any agency thereof. The views and opinions of authors expressed herein do not necessarily state or reflect those of the United States Government or any agency thereof.

\section{Availability}

This report is posted on the U.S. Department of Energy's Princeton Plasma Physics Laboratory Publications and Reports web site in Fiscal Year 2003. The home page for PPPL Reports and Publications is: http://www.pppl.gov/pub_report/

DOE and DOE Contractors can obtain copies of this report from:

U.S. Department of Energy

Office of Scientific and Technical Information

DOE Technical Information Services (DTIS)

P.O. Box 62

Oak Ridge, TN 37831

Telephone: (865) 576-8401

Fax: (865) 576-5728

Email: reports@adonis.osti.gov

This report is available to the general public from:

National Technical Information Service

U.S. Department of Commerce

5285 Port Royal Road

Springfield, VA 22161

Telephone: $1-800-553-6847$ or

(703) $605-6000$

Fax: (703) 321-8547

Internet: http://www.ntis.gov/ordering.htm 


\title{
Destabilization of fast magnetoacoustic waves by circulating energetic ions in toroidal plasmas
}

\author{
V.S. Belikov ${ }^{1}$, Ya. I. Kolesnichenko ${ }^{1}$, R. B. White ${ }^{2}$ \\ ${ }^{1}$ Institute for Nuclear Research, Prospekt Nauky 47, Kyiv 03680, Ukraine \\ 2 Princeton Plasma Physics Laboratory, P.O. Box 451, Princeton, NJ 08543, USA
}

(July 28, 2003)

\begin{abstract}
An instability of fast magnetoacoustic waves (FMW) driven by circulating energetic ions in axisymmetric toroidal plasmas and characterized by the frequencies below the ion gyrofrequency is considered. An important role of the $l=0$ resonance ( $l$ is the number of a cyclotron harmonic) in the waveparticle interaction is revealed: It is shown that this resonance considerably extends an unstable region in the space of the pitch-angles of the energetic ions and the wave frequencies. The analysis is carried out for a "slow" instability, which has the growth rate less than the bounce frequency of the energetic ions. Specific examples relevant to the National Spherical Torus Experiment (NSTX) [J. Spitzer et al., Fusion Technol. 30, 1337 (1996)], where instabilities of this kind were observed, are considered.
\end{abstract}




\section{INTRODUCTION}

The instability of Fast Magnetoacoustic Waves (FMW) caused by the energetic ions was first considered in 70s, see, e.g., an overview. ${ }^{1}$ Later it was found that it can be responsible for the superthermal Ion Cyclotron Emission (ICE) with the frequencies $\omega \geq$ $\omega_{B}$ ( $\omega_{B}$ is the ion gyrofrequency) observed in experiments on tokamaks. ${ }^{2}$ This stimulated further development of theory of the FMW instability. In particular, it was found that the presence of a drift term in the local cyclotron resonance condition plays an important role by strongly enhancing the instability; moreover, it may explain a number of peculiarities of the ICE frequency spectrum in Joint European Torus $(\mathrm{JET})^{3}$ experiments. $^{4,5}$ This result was obtained for a "rapid" instability, i.e., the instability with the growth rate, $\gamma$, exceeding the bounce/transit frequency of the energetic ions, $\omega_{b}$. The mentioned condition may be not satisfied, in which case "slow" instability $\left(\gamma<\omega_{b}\right)$ takes place. ${ }^{6,7}$ In more recent time, a low-frequency FMW instability, $\omega<\omega_{B}$, was observed in National Spherical Torus $(\mathrm{NSTX})^{8}$ experiments. ${ }^{9,10}$ This instability seems to contribute to the bulk ion heating due to a non-linear mechanism leading to stochastic motion of the particles. ${ }^{11} \mathrm{~A}$ linear theory of "slow" FMW instability with $\omega<\omega_{B}$ was developed in Ref. ${ }^{12}$. The instability considered in the mentioned work is driven through the cyclotron resonance with $l=1$, where $l$ is the number of the cyclotron harmonic. It was claimed in Ref. ${ }^{12}$ that the $l=0$ resonance cannot lead to the instability. However, this statement is not correct, which follows from the present work, where we develop a theory including the influence of the $l=0$ resonance on the destabilization of FMW. In numerical examples we use NSTX parameters.

The paper is organized as follows. In Sec. II the resonances of the waves and circulating energetic ions are considered, a qualitative analysis which demonstrates a possible excitation of the $l=0$ instability (with $\gamma<\omega_{b}$ or $\gamma>\omega_{b}$ ) is carried out. In Sec. III the growth rate of the "slow" FMW instability is calculated and analyzed. In Sec. IV the obtained results are summarized and their consistency with experimental observations of FMW in NSTX is discussed. In Appendix the anti-Hermitian part of the flux-surface 
averaged dielectric permeability tensor of well circulating particles is calculated in the assumption that characteristic times of interest exceed the particle transit time.

\section{QUALITATIVE ANALYSIS}

In order to see that the $l=0$ resonance can be of importance, we note that the instability drive is proportional to $d F_{b} / d \mathbf{v}$, where $F_{b}$ is the equilibrium distribution function of the energetic ions, $\mathbf{v}$ is the particle velocity. The case of $l=0$ corresponds to neglecting the particle Larmor rotation. In this case, when $F_{b}=F_{b}(\mathcal{E}, \mu)(\mathcal{E}$ and $\mu$ are the particle energy and the magnetic moment, respectively), $d F_{b} / d \mathbf{v}=M \mathbf{v} \partial F_{b} / \partial \mathcal{E}$ with $M$ the beam ion mass, which implies that the instability drive is absent unless $F_{b}(\mathcal{E})$ is a non-monotonic function. Taking into account the Larmor rotation results in a term proportional to $l \partial F_{b} / \partial \mu$, which drives the instability considered in Ref. ${ }^{12}$. However, the distribution function of the energertic ions typically depends on $\lambda \equiv \mu B_{0} / \mathcal{E}$ rather than on $\mu$ (because $\lambda$ is approximately conserved during the collisional slowing down of the ions when $\mathcal{E} \gg \mathcal{E}_{c}$ with $\mathcal{E}_{c} \sim\left(M / M_{e}\right)^{1 / 3} T_{e}, T_{e}$ and $M_{e}$ the electron temperature and mass, respectively). Therefore, a term associated with the velocity anisotropy appears even when effects of Larmor rotation are neglected (the $l=0$ case): $d F_{b} / d \mathbf{v}=M \mathbf{v}\left[\partial F_{b} / \partial \mathcal{E}-\lambda \mathcal{E}^{-1} \partial F_{b} / \partial \lambda\right]$.

The physical mechanism responsible for the "slow" instability is actually the same as that of the "rapid" instability: both instabilities are driven by the velocity anisotropy of the energetic ions through the "local" resonance:

$$
\omega-l \omega_{B}=k_{\|} v_{\|}+\omega_{d}
$$

where $k_{\|}=(m-n q) /\left(q R_{0}\right)$ is the longitudinal mode number, $m$ and $n$ are the poloidal and toroidal mode numbers, respectively, $q$ is the safety factor, $R_{0}$ is the radius of the magnetic axis, $v_{\|}$is the longitudinal velocity of the energetic ions, $\omega_{d}=\mathbf{k} \cdot \mathbf{v}_{\mathbf{d}}, \mathbf{v}_{\mathbf{d}}$ is the particle drift velocity, $\mathbf{k}$ is the wave vector. Equation (1) is written in the assumption that the perturbed quantities are proportional to $\exp (-i \omega t+i m \vartheta-i n \varphi)$, where $\vartheta$ is the poloidal angle, $\varphi$ is the toroidal angle. Because the mode is localized at the outer midplane 
of the torus, ${ }^{7}$ the last term in Eq. (1) is reduced to $\omega_{d} \approx-m v_{d} / r$ where $r$ is the radial coordinate. If the resonance condition is satisfied, the particle drive is maximum for the particles with the highest energy. For this reason, Eq. (1) can be considered as an equation which determines the mode numbers of the destabilized waves. If so, we can conclude that the $l=0$ resonance extends the frequency spectrum of the instability driven through $l \neq 0$ resonances. This fact is of importance to lower the threshold magnitudes of the wave amplitudes required for stochastic heating on sub-harmonics of the ion gyrofrequency. ${ }^{11}$

The growth rates of both "slow" and "rapid" instabilities can be expressed through Bessel functions of the $l^{\text {th }}$ order, $J_{l}(\xi)$, where $\xi=k_{\perp} \rho_{\perp}=\left(\omega / \omega_{B}\right) \sqrt{\lambda} v / v_{A}, \rho_{\perp}=v_{\perp} / \omega_{B}$, $v_{\perp}$ is the particle velocity across the magnetic field. The $l \neq 0$ instability can occur only when $\xi>1$, which is difficult to satisfy for well circulating particles $(\lambda \ll 1)$ when $\omega \ll \omega_{B}$. In contrast to this, as will be shown in this work, the $l=0$ instability exists even when $\xi<1$. This implies that it can be driven by particles with larger pitch angles (smaller $\lambda$ ) and, in addition, it can have lower wave frequencies.

For the "slow" instability to occur, an additional condition of the "global" resonance between the particles and the waves must be satisfied. This condition can be written as

$$
\omega-l\left\langle\omega_{B}\right\rangle=m \omega_{\vartheta}-n \omega_{\varphi}+s \omega_{b}
$$

where $s$ is an integer, $\langle\ldots\rangle$ denotes the transit time averaging, $\omega_{\vartheta}$ and $\omega_{\varphi}$ are the frequencies of the particle poloidal rotation and toroidal rotation, respectively. Equation (2) is satisfied only for certain magnitudes of $s$. In order to evaluate $s$ we specify the pitch angles of the energetic ions. We assume that the population of the energetic ions with the energy close to their maximum energy, $\mathcal{E}_{0}$, consists mainly of the well circulating particles. Then $\omega_{b}=\left|\omega_{\vartheta}\right| \approx\left|\left\langle v_{\|}\right\rangle / q R_{0}\right|$, and we can write Eq. (2) in the following form:

$$
\omega-l\left\langle\omega_{B}\right\rangle=k_{\|}\left\langle v_{\|}\right\rangle+s \frac{\left\langle v_{\|}\right\rangle}{q R_{0}},
$$

Comparing Eq. (1) and Eq. (3) we conclude that

$$
s \frac{\left\langle\rho_{\|}\right\rangle}{q R_{0}} \approx \frac{\omega_{d}}{\left\langle\omega_{B}\right\rangle}+l \frac{\omega_{B}-\left\langle\omega_{B}\right\rangle}{\left\langle\omega_{B}\right\rangle},
$$


where $\rho_{\|}=v_{\|} /\left\langle\omega_{B}\right\rangle$. It follows from Eq. (4) that the sign of $s$ is determined by the sign of $m$ for $l=0$ and for $l \neq 0$ when the $\omega_{d} /\left\langle\omega_{B}\right\rangle$ is large enough. Another conclusion is that the presence of the $\omega_{d}$ term in Eq. (1) reconciles the condition of the local resonance with the condition of the global one for circulating particles when $l=0$.

Let us consider a specific example relevant to an NSTX plasma. We take $R_{0}=100$ $\mathrm{cm}$, the Alfvén velocity $v_{A}=10^{8} \mathrm{~cm} \mathrm{~s}^{-1}$, the particle injection energy $\mathcal{E}_{0}=80 \mathrm{keV}$, $\omega_{B}=1.5 \times 10^{7} \mathrm{~s}^{-1}, \rho \equiv v / \omega_{B}=20 \mathrm{~cm}$. Then $\omega_{d} /\left\langle\omega_{B}\right\rangle=0.6 \widetilde{\omega} / \kappa$ with $\widetilde{\omega}=\omega /\left\langle\omega_{B}\right\rangle$ and $\kappa$ the elongation of the plasma cross-section. The second term in the right-hand side of Eq. (4) can be evaluated as $l \epsilon$ with $\epsilon=r / R_{0}$, i.e., it slightly exceeds the $\omega_{d} /\left\langle\omega_{B}\right\rangle$ term. This implies that the magnitudes of $s$ satisfying Eq. (4) are different for $l=0$ and $l=1$. For $l=0$ we obtain $|s| \leq 3 q \chi^{-1} \widetilde{\omega} / \kappa$ (we used $v_{0} / v_{A}=3$, where $v_{0}=\left(2 \mathcal{E}_{0} / M\right)^{1 / 2}$ ), i.e., $|s| \sim 1$ for $\widetilde{\omega} \lesssim 1 / 2, q \sim 2$

Note that when $\omega<\omega_{B}$, typically the global resonance condition can be satisfied for $l \geq 0$, but not for $l<0$. Indeed, it follows from Eq. (3) that

$$
\frac{k}{k_{\| s}} \frac{v_{A}}{v_{0} \sqrt{1-\lambda}}\left(1-\frac{l}{\widetilde{\omega}}\right)<1,
$$

where $k_{\| s}=k_{\|}+s /(q R)$ and $k / k_{\| s}>1$ [we used $k \approx \omega / v_{A}=\widetilde{\omega}\left(v_{0} / v_{A}\right) \rho^{-1} \gg s /\left(q R_{0}\right)$ ].

\section{GROWTH RATE OF THE INSTABILITY}

Assuming $k_{\|} \ll k_{\perp}$, we proceed from the following dispersion relation for FMW in a plasma with energetic ions: ${ }^{13}$

$$
\Lambda(\omega) \equiv \bar{\varepsilon}_{11}\left(\bar{\varepsilon}_{22}-N_{\perp}^{2}\right)+\bar{\varepsilon}_{12}^{2}=0,
$$

where $N_{\perp}=c k_{\perp} / \omega, \bar{\varepsilon}_{i j}$ are the flux-surface-averaged components of the dielectric tensor. We treat the problem perturbatively, in which case the contribution of the energetic ions to the Hermitian part of the permeability tensor, $\bar{\varepsilon}_{i j}^{\prime}$, can be neglected, and we have: ${ }^{14}$

$$
\bar{\varepsilon}_{11}^{\prime}=\bar{\varepsilon}_{22}^{\prime}=\frac{\omega_{p i}^{2}}{\omega_{B i}^{2}-\omega^{2}}
$$




$$
\bar{\varepsilon}_{12}^{\prime}=-\bar{\varepsilon}_{21}^{\prime}=i \bar{g}^{\prime}, \quad \bar{g}^{\prime}=\frac{\omega}{\omega_{B i}} \bar{\varepsilon}_{11}^{\prime}
$$

where $\omega_{B i}$ and $\omega_{p i}$ are the gyrofrequency and the plasma frequency of the bulk plasma ions, respectively. Note that Eq. (8) for the considered waves with $\omega<\omega_{B i}$ is valid for both a "cold" plasma ( $\omega \gg k_{\|} v_{t h, i}, \omega \gg k_{\|} v_{t h, e}$, where $v_{t h}$ is the thermal velocity) and a plasma with "cold" ions and "hot" electrons $\left(\omega \ll k_{\|} v_{t h, e}\right)$. Assuming that the antiHermitian part of the dielectric permeability tensor, $\bar{\varepsilon}_{i j}^{\prime \prime}$, is small, we can write $\omega=\omega_{0}+i \gamma$, where $\omega_{0} \gg \gamma$. Then Eq. (6) yields $\omega_{0}=k_{\perp} v_{A}$ and

$$
\gamma=-\frac{\bar{\varepsilon}_{11}^{\prime \prime}\left(\bar{\varepsilon}_{22}^{\prime}-N_{\perp}^{2}\right)+\bar{\varepsilon}_{22}^{\prime \prime} \bar{\varepsilon}_{11}^{\prime}-2 \bar{g}^{\prime \prime} \bar{g}^{\prime}}{\partial \Lambda^{\prime} / \partial \omega}
$$

where the subscript "0" at $\omega$ is omitted. Components of the Hermitian part of the dielectric tensor can be eliminated from Eq. (9) due to Eqs. (6) - (8). As a result, Eq. (9) is reduced to

$$
\frac{\gamma}{\omega}=-\frac{v_{A}^{2}}{2 c^{2}}\left(\bar{\varepsilon}_{22}^{\prime \prime}-2 \frac{\omega}{\omega_{B i}} \bar{g}^{\prime \prime}+\frac{\omega^{2}}{\omega_{B i}^{2}} \bar{\varepsilon}_{11}^{\prime \prime}\right)
$$

Let us neglect the wave damping caused by the bulk plasma and assume that the population of the energetic ions consists only of circulating particles. Then can use the expressions for $\bar{\varepsilon}_{i j}^{\prime \prime}$ obtained in Appendix. This leads to

$$
\frac{\gamma}{\omega}=\left.2 \pi^{2} \frac{\omega_{p b}^{2}}{k_{\perp}^{2} c^{2}} \sum_{l, s, \sigma} \int \frac{d \lambda \lambda \mathcal{E}^{2}}{M\left|k_{\| s}\right|(1-\lambda)} Q_{l}{ }^{2}(\xi) J_{s}{ }^{2}(\zeta) \hat{\Pi}_{l} F_{b}(\mathcal{E}, \lambda)\right|_{\mathcal{E}=\mathcal{E}_{s}}
$$

where $l$ and $s$ are integers, $\sigma=\operatorname{sgn} v_{\|}$,

$$
\begin{aligned}
\mathcal{E}_{s} & =\frac{M v_{\| s}^{2}}{2(1-\lambda)}, \quad v_{\| s}=\frac{\omega-l \omega_{B 0}}{k_{\| s}}, \quad k_{\| s}=k_{\|}+\frac{s}{q R} \\
Q_{l}(\xi) & =J_{l}^{\prime}(\xi)-\frac{\omega}{\omega_{B i}} \frac{l}{\xi} J_{l}(\xi), \quad \hat{\Pi}=\omega \frac{\partial}{\partial \mathcal{E}}+\left(l \omega_{B 0}-\lambda \omega\right) \frac{\partial}{\mathcal{E} \partial \lambda}
\end{aligned}
$$

and $\xi=k_{\perp} v_{s} \sqrt{\lambda} / \omega_{B 0}, \zeta$ is defined in Appendix, $\left.\omega_{B 0}=\left\langle\omega_{B}\right\rangle(\lambda=0)\right)$. Note that we used the condition of the "global" resonance given by Eq. (3) in order to integrate over $\mathcal{E}$ in Eq. (11).

Now we have to specify the distribution function of the energetic ions. We take it in the form: 


$$
F_{b}(\mathcal{E}, \lambda)=f_{\mathcal{E}}(\mathcal{E}) \delta\left(\lambda-\lambda_{0}\right) \eta\left(\mathcal{E}_{0}-\mathcal{E}\right) \eta\left(\mathcal{E}-\mathcal{E}_{c}\right)
$$

where $\lambda_{0}$ and $\mathcal{E}_{0}$ are the initial pitch-angle parameter and energy of the beam ions, $\eta(x)=$ $\int_{-\infty}^{x} \delta(x) d x, \delta(x)$ is the Dirac delta function.

One can see that when $\lambda$ is sufficiently small, the $\lambda$ dependence can be neglected in $J_{s}(\zeta)$. Then, after integration over $\lambda$ in Eq. (11) we obtain:

$$
\begin{array}{r}
\frac{\gamma_{b}}{\omega}=2 \pi^{2} \frac{\omega_{p b}^{2}}{k_{\perp}^{2} c^{2}} \sum_{l, s} \frac{\omega}{\left|k_{\| s}\right|} \frac{\mathcal{E}_{b}}{M} J_{s}^{2}\left(\zeta_{b}\right)\left\{\lambda_{0} Q_{l}^{2}\left(\xi_{b}\right) \frac{d}{d \mathcal{E}_{b}}\left(\mathcal{E}_{b} f_{\mathcal{E}}\left(\mathcal{E}_{b}\right)\right)\right. \\
\left.+\left(\lambda_{0}-\frac{l \omega_{B 0}}{\omega}\right) \frac{f_{\mathcal{E}}\left(\mathcal{E}_{b}\right)}{2 \xi_{b}} \frac{d}{d \xi_{b}}\left(\xi_{b}^{2} Q_{l}^{2}\left(\xi_{b}\right)\right)\right\}
\end{array}
$$

where

$$
\begin{gathered}
v_{c}<\left|v_{\| s}\right| / \sqrt{1-\lambda_{0}}<v_{0}, \\
\mathcal{E}_{b}=\frac{M v_{\| s}^{2}}{2\left(1-\lambda_{0}\right)}, \quad \xi_{b}=\frac{k_{\perp} v_{\| s}}{\omega_{B 0}}\left(\frac{\lambda_{0}}{1-\lambda_{0}}\right)^{1 / 2}, \\
\zeta_{b}=\left(\zeta_{\| b}^{2}+\zeta_{\perp b}^{2}\right)^{1 / 2}, \quad \zeta_{\| b}=\frac{l \omega_{B 0} q r}{v_{\| s}}, \quad \zeta_{\perp b}=\frac{k_{\perp} q v_{\| s}}{\omega_{B 0} \kappa} \frac{1-\lambda_{0} / 2}{1-\lambda_{0}} .
\end{gathered}
$$

Finally, taking $f_{\mathcal{E}}=C / \mathcal{E}^{3 / 2}$ with $C=\sqrt{1-\lambda_{0}} M^{3 / 2} /\left(\pi \sqrt{2} \ln \left(\mathcal{E}_{0} / \mathcal{E}_{c}\right)\right)$ we have:

$$
\begin{aligned}
\frac{\gamma_{b}}{\omega}=C \pi^{2} \frac{\omega_{p b}^{2}}{k_{\perp}^{2} c^{2}} \sum_{l, s} \frac{\omega}{\left|k_{\| s}\right|}\left(\frac{M}{\mathcal{E}_{b}}\right)^{1 / 2} & J_{s}^{2}\left(\zeta_{b}\right)\left\{\lambda_{0} \frac{d}{d \xi_{b}}\left(\xi_{b} Q_{l}^{2}\left(\xi_{b}\right)\right)\right. \\
& \left.-\frac{l \omega_{B 0}}{\omega} \frac{1}{\xi_{b}} \frac{d}{d \xi_{b}}\left(\xi_{b}^{2} Q_{l}^{2}\left(\xi_{b}\right)\right)\right\} .
\end{aligned}
$$

It follows from Eq. (17) that a necessary condition of the instability is

$$
D_{l} \equiv \frac{d}{d \xi_{b}}\left(\xi_{b} Q_{l}\left(\xi_{b}\right)\right)-\frac{l}{\lambda_{0}} \frac{\omega_{B 0}}{\omega} \frac{1}{\xi_{b}} \frac{d}{d \xi_{b}}\left(\xi_{b}^{2} Q_{l}\left(\xi_{b}\right)\right)>0
$$

which must be must be satisfied, at least, for some $l$ and $s$. Another necessary condition given by Eq. (15) can be written as

$$
v_{c} \sqrt{1-\lambda_{0}}<\left|\frac{\omega-l \omega_{B 0}}{k_{\| s}}\right|<v_{0} \sqrt{1-\lambda_{0}} .
$$

Note that the right inequality in Eq. (19) is actually Eq. (5).

The functions $D_{0}$ and $D_{1}$ are shown in Fig. 1 . We observe that the condition $D_{0}>0$ is satisfied for $\xi_{b}>0$, which implies that it is satisfied for arbitrary small $\lambda_{0}$. In contrast to 
this, $D_{1}>0$ only when $\xi_{b}$ exceeds a certain magnitude $\xi_{\text {min }}$. The latter weakly depends on $\omega$, being a decreasing function. More detailed analysis shows that $D_{1}$ almost does not depend on $\lambda_{0}$. Using these facts and that $\xi_{b} \widetilde{\omega}\left(v_{\|} / v_{A}\right) \sqrt{\lambda_{0} /\left(1-\lambda_{0}\right)}<\widetilde{\omega}\left(v_{\|} / v_{A}\right) \sqrt{\lambda_{0}}$, we obtain the following condition for the $l=1$ driven instability:

$$
\lambda_{0} \widetilde{\omega}^{2}>\left(\xi_{\min } \frac{v_{A}}{v_{0}}\right)^{2} .
$$

Equation (20) shows that $\omega$ cannot be arbitrary small. On the other hand, for a given $\widetilde{\omega}$, this equation gives a restriction for $\lambda_{0}$. For instance, when $v_{0} / v_{A}=3$, it cannot be satisfied for $\widetilde{\omega} \lesssim 0.3$, and it yields $\lambda>0.3$ for $\widetilde{\omega}=0.5$. We conclude from here that only the $l=0$ instability is possible for sufficiently small $\widetilde{\omega}$. In the other case, $\widetilde{\omega} \rightarrow 1$, Eq. (20) can be satisfied small $\lambda$, but, nevertheless, the $l=1$ instability is absent or weak because then $D_{l} \rightarrow 0$, see Fig. 1 .

Now we calculate the growth rate for various directions of the wave propagation using Eq. (17). The results for co-injection (which corresponds to NSTX experiments) are shown in Figs. 2, 3. We observe that the growth rate of the instability with $k_{\|}>0$ considerably exceeds that one for $k_{\|}<0$. The reason for this is that the $l=0$ resonance, which provides the strongest drive (see Fig. 1), is responsible for the instability in the first case, whereas the $l=1$ resonance leads to the instability in the second one (the $l=0$ resonance takes place for $k_{\| s}>0$, which leads to $k_{\|}>0$ when $\left.R_{0} / \rho>v_{0} / v_{A}\right)$. Note that the non-monotonic dependence of $\gamma$ on $\lambda_{0}$ shown in Fig. 2 is caused by the fact that the number of the terms in the sum over $s$ in Eq. (17) depends on $\lambda_{0}$.

\section{SUMMARY AND CONCLUSIONS}

Our analysis shows that the $l=0$ resonance of the wave-particle interaction provides the destabilization of FMW with the frequencies lower than those destabilized through the $l=1$ resonance. In addition, it makes possible the destabilization of the waves by the energetic ions with smaller $\lambda$ (larger pitch angles).

These facts are of importance for the interpretation of NSTX experimental data reported in Ref. ${ }^{9}$. In the mentioned experiments the modes in the frequency range $0.4-2.5$ 
MHz were observed. This means that the lowest frequency, $\omega_{\min }$, was about $\omega_{B} / 6$, which cannot be explained by theory ignoring the $l=0$ resonance. Another important experimental fact is that the mode excitation is sensitive to NBI injection angle: the switch in the injection angle accompanied by the decrease of the number of circulating particles resulted in stabilization of modes with lowest frequencies, which was observed in the shot \#104505. ${ }^{9}$ This fact can be explained by our theory, which predicts that the instability exists only when $k_{\perp} \rho_{\perp}=\sqrt{\lambda_{0}}\left(\omega / \omega_{B}\right)\left(v_{0} / v_{A}\right)<2$, and thus, the increase of $\lambda_{0}$ may violate the latter condition. In addition, our consideration agrees with the experimentally observed reduction of the number of unstable modes when switching the beam energy from $\mathcal{E}_{0}=80 \mathrm{keV}$ to $\mathcal{E}_{0}=70 \mathrm{keV}$ with the same injection power ${ }^{12}$ : It follows from the obtained expressions that $\gamma_{b} \sim J_{1}{ }^{2}\left(\xi_{b}\right) J_{s}{ }^{2}\left(\zeta_{b}\right) / \sqrt{\mathcal{E}_{0}} \sim \mathcal{E}_{0}{ }^{s+1 / 2}$, therefore, the decrease of $\mathcal{E}_{0}$ may result in violation of the instability condition $\gamma_{b}>\gamma_{d}$, where $\gamma_{d}$ is the mode damping.

Thus, the carried out analysis reveals an important role of the $l=0$ resonance. On the other hand, the mentioned resonance is possible due to the presence of the drift term in the local resonance condition, as it follows from Eq. (4). Therefore, the conclusion that the toroidal drift enhances the destabilization of FMW, which was made for the "rapid" high-frequency $\left(\omega>\omega_{B}\right)$ instability in Refs. ${ }^{4,5}$, is valid also for the "slow" instability with $\omega<\omega_{B}$. This indicates physical mechanisms responsible for "slow" and "fast" instabilities are similar, the mentioned instabilities are essentially the same FMW instability, which, depending on conditions, has the growth rate either larger or less than the transit frequency of the energetic ions.

\section{ACKNOWLEDGEMENTS}

The research described in this publication was made possible in part by the Award No. UP2-2419-KV-02 of the U.S. Civilian Research \&Development Foundation and the Government of Ukraine, and the US Department of Energy Grant DE-FG03-94ER54271. 


\section{APPENDIX A: ANTI-HERMITIAN PART OF THE DIELECTRIC PERMEABILITY TENSOR OF CIRCULATING IONS}

We proceed from the following general expression for the dielectric permeability tensor in local approximation ${ }^{14}$

$$
\begin{aligned}
\varepsilon_{i j}=\delta_{i j}- & i \frac{\omega_{p}^{2}}{\omega} \int d \mathbf{v} v_{i}(t) \int_{-\infty}^{t} \frac{\partial F}{\partial v_{l}\left(t^{\prime}\right)}\left\{\left(1-\frac{\mathbf{k} \mathbf{v}\left(t^{\prime}\right)}{\omega}\right) \delta_{l j}\right. \\
+ & \left.\frac{k_{l} v_{j}\left(t^{\prime}\right)}{\omega}\right\} \exp \left\{i \omega\left(t-t^{\prime}\right)-i \mathbf{k} \int_{t^{\prime}}^{t} \mathbf{v}\left(t^{\prime \prime}\right) d t^{\prime \prime}\right\}
\end{aligned}
$$

where the time integration is carrying out along the unperturbed orbit, $F$ the equilibrium distribution function.

We follow the approach of Ref. ${ }^{15}$, assuming that characteristic times exceed the particle transit time. Then we obtain the anti-Hermitian part of the dielectric permeability tensor averaged over the flux surfaces in the form:

$$
\bar{\varepsilon}_{i j}^{\prime \prime}=-\pi \frac{\omega_{p}^{2}}{\omega^{2}} \sum_{l, s, \sigma} \int \mathcal{E} d \mathcal{E} d \lambda \frac{\tau_{b}}{M q R} q_{i} q_{j}\left|G_{l, s}\right|^{2} \delta\left(\left\langle\Omega_{l}\right\rangle-s \omega_{b}\right) \hat{\Pi}_{l} F(\mathcal{E}, \lambda),
$$

where $\tau_{b}$ is the particle transit time, $l$ and $s$ are integers, $\sigma=\operatorname{sgn} v_{\|}, \mathcal{E}=M v^{2} / 2$, $\lambda=\mu B_{0} / \mathcal{E}, \mu=M v_{\perp}^{2} / 2 B$,

$$
\begin{aligned}
\hat{\Pi}_{l} & =\omega \frac{\partial}{\partial \mathcal{E}}+\left(l \omega_{B 0}-\lambda \omega\right) \frac{\partial}{\mathcal{E} \partial \lambda}, \quad \int F(\mathbf{v}) d \mathbf{v}=1 \\
\mathbf{q} & =\left\{v_{\perp} \frac{l}{\xi} J_{l}(\xi), i v_{\perp} J_{l}^{\prime}(\xi)\right\}, \quad \xi=\frac{k_{\perp} v_{\perp}}{\omega_{B 0}}, \\
G_{l, s} & =\left\langle\exp \left\{i W(t)-i s \omega_{b} t\right\}\right\rangle, \\
W(t) & =\int_{0}^{t}\left(l\left(\omega_{B}-\left\langle\omega_{B}\right\rangle\right)+k_{\|}\left(v_{\|}-\left\langle v_{\|}\right\rangle\right)+\omega_{d}-\left\langle\omega_{d}\right\rangle\right) d t^{\prime}, \\
\left\langle\Omega_{l}\right\rangle & =\omega-l\left\langle\omega_{B}\right\rangle-k_{\|}\left\langle v_{\|}\right\rangle-\left\langle\omega_{d}\right\rangle, \quad\langle\ldots\rangle=\frac{1}{\tau_{b}} \int_{0}^{\tau_{b}} d t(\ldots)
\end{aligned}
$$

where $J_{l}$ is the $l$ th order Bessel function. In the small-orbit-width approximation, $\mid r-$ $r_{0} \mid \ll r$, the expression for $G_{l, s}$ for the well circulating particles, is reduced to: ${ }^{15}$

$$
G_{l, s}=J_{s}(\zeta)
$$

where 


$$
\begin{aligned}
\zeta & =\left(\zeta_{\|}^{2}+\zeta_{\perp}^{2}\right)^{1 / 2}, \quad \zeta_{\|}=\frac{l \omega_{B 0} q r}{v_{\| 0}}, \quad \zeta_{\perp}=k_{\perp} q R \frac{v_{d}}{v_{\| 0}} \\
v_{\| 0} & =\sigma v(1-\lambda)^{1 / 2}, \quad v_{d}=\frac{v^{2}(1-\lambda / 2)}{\omega_{B 0} \kappa R} \\
\left\langle\Omega_{l}\right\rangle & =\omega-l \omega_{B 0}-k_{\|} v_{\| 0}, \quad \omega_{b}=v_{\| 0} /(q R),
\end{aligned}
$$

where $\omega_{B 0}=\left\langle\omega_{B}\right\rangle(\lambda=0), \kappa$ the elongation of the plasma cross section. 


\section{References}

${ }^{1}$ Ya. I. Kolesnichenko, Nucl. Fusion 20, 727 (1980).

${ }^{2}$ G. A. Cottrell, R. O. Dendy, Phys. Rev. Lett. 60, 33 (1988).

${ }^{3}$ JET Team, Nucl. Fusion 32, 187 (1992).

${ }^{4}$ Ya. I. Kolesnichenko, D. Anderson, T. Fülöp, M. Lisak, Fusion Energy 1996 (Proc. 16th IAEA Conference on Fusion Energy, Montreal, Canada), IAEA, Vienna, v.2, 497 (1997).

${ }^{5}$ T. Fülöp, Ya.I. Kolesnichenko, M. Lisak, D. Anderson, Nucl. Fusion 37, 1281 (1997).

${ }^{6}$ V. S. Belikov, Ya. I. Kolesnichenko, O. A. Silivra, Nucl. Fusion 35, 1603 (1995).

${ }^{7}$ N. N. Gorelenkov, C. Z. Cheng, Phys. Plasmas 2, 1961 (1995).

${ }^{8}$ J. Spitzer, M. Ono, M. Peng et al., Fusion Technol. 30, 1337 (1996).

${ }^{9}$ E. D. Fredrickson, N. N. Gorelenkov, C. Z. Cheng et al., Phys. Rev. Lett. 87, 145001 (2001).

${ }^{10}$ D. A. Gates, N. N. Gorelenkov, R. B. White, Phys. Rev. Lett. 87, 205003 (2001).

${ }^{11}$ L. Chen, Z. Lin, and R. White, Phys. Plasmas 8, 4713 (2001).

${ }^{12}$ N. N. Gorelenkov, C. Z. Cheng, F. Fredrickson et al., Nucl. Fusion, 42, 977 (2002).

${ }^{13}$ T. D. Kaladze, A. B. Mikhajlovskij, Sov. J. Plasma Phys 1, 128 (1975).

${ }^{14}$ V. D. Shafranov, in Reviews of Plasma Pysics, edited by M. A. Leontovich (Consultants Bureau, New York, 1967), Vol. 3, p. 3.

${ }^{15}$ V. S. Belikov, Ya. I. Kolesnichenko, Plasma Physics 24, 61 (1982). 


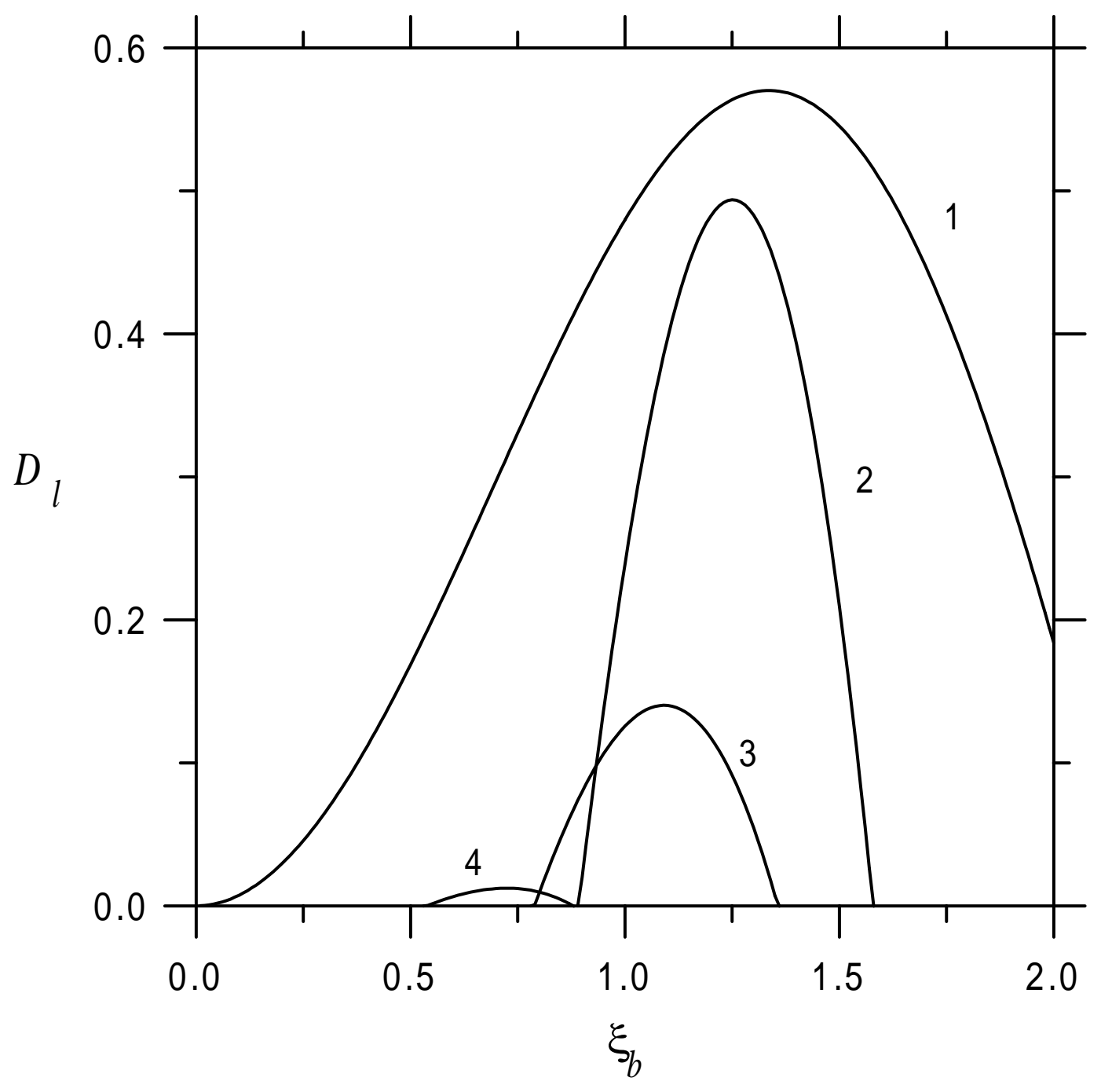

FIG. 1. Dependence of the growth rate drive, $D_{l}$, on $\xi_{b}$ for $\lambda_{0}=0.4 .1, l=0 ; 2, l=1$ and $\widetilde{\omega}=0.3 ; 3, l=1$ and $\widetilde{\omega}=0.5 ; 4, l=1$ and $\widetilde{\omega}=0.8$. 


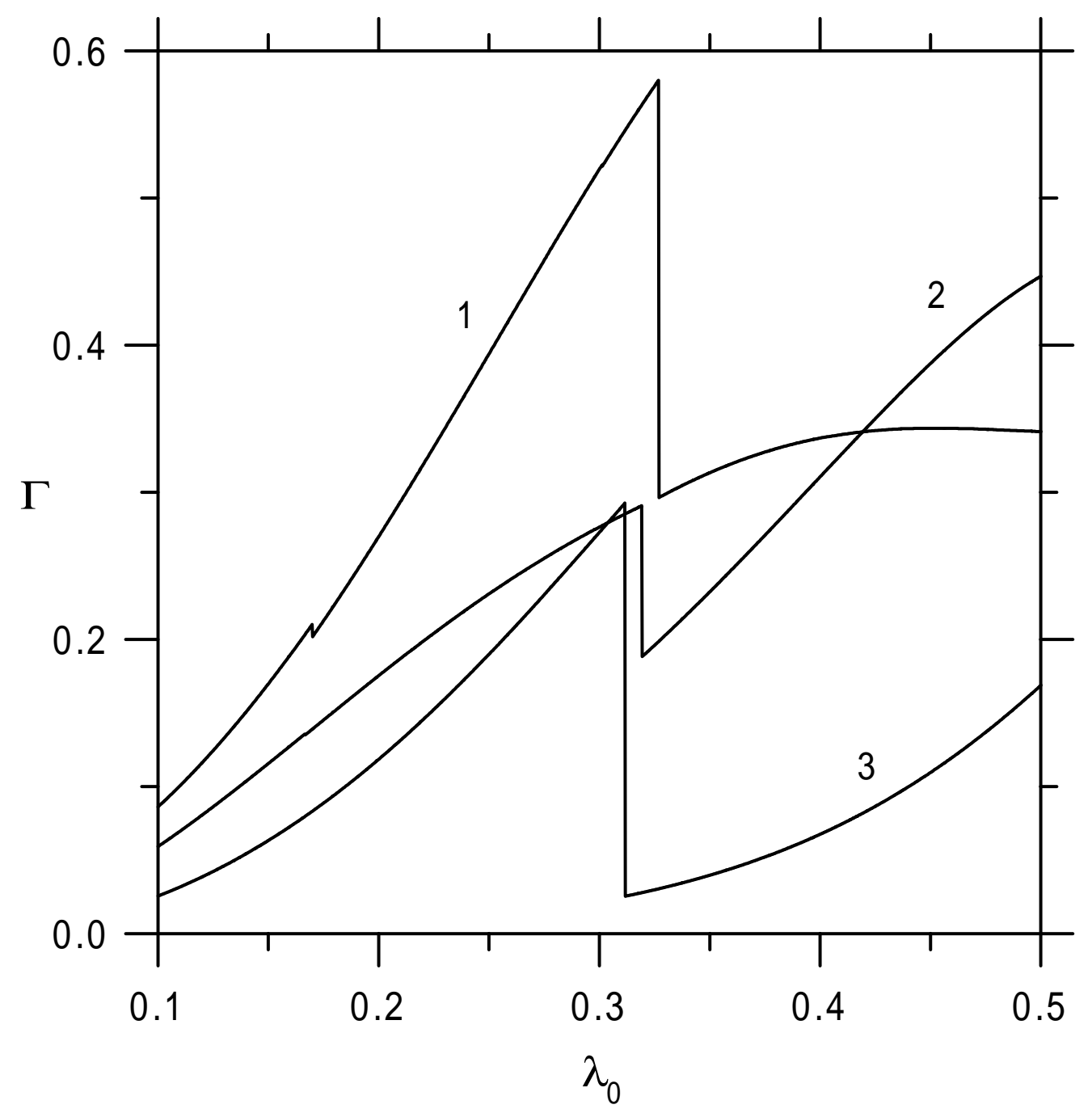

FIG. 2. Normalized growth rate, $\Gamma=\left(\gamma_{b} / \omega\right)\left(n / n_{b}\right)$, versus the pitch-angle parameter for $k_{\|}>0, \widetilde{\omega}=0.5$, and various directions of the wave propagation: $1,\left|k_{\|}\right| / k=0.5 ; 2,\left|k_{\|}\right| / k=0.4$; $3,\left|k_{\|}\right| / k=0.3$. Here $n$ and $n_{b}$ are the plasma and beam density, respectively. 


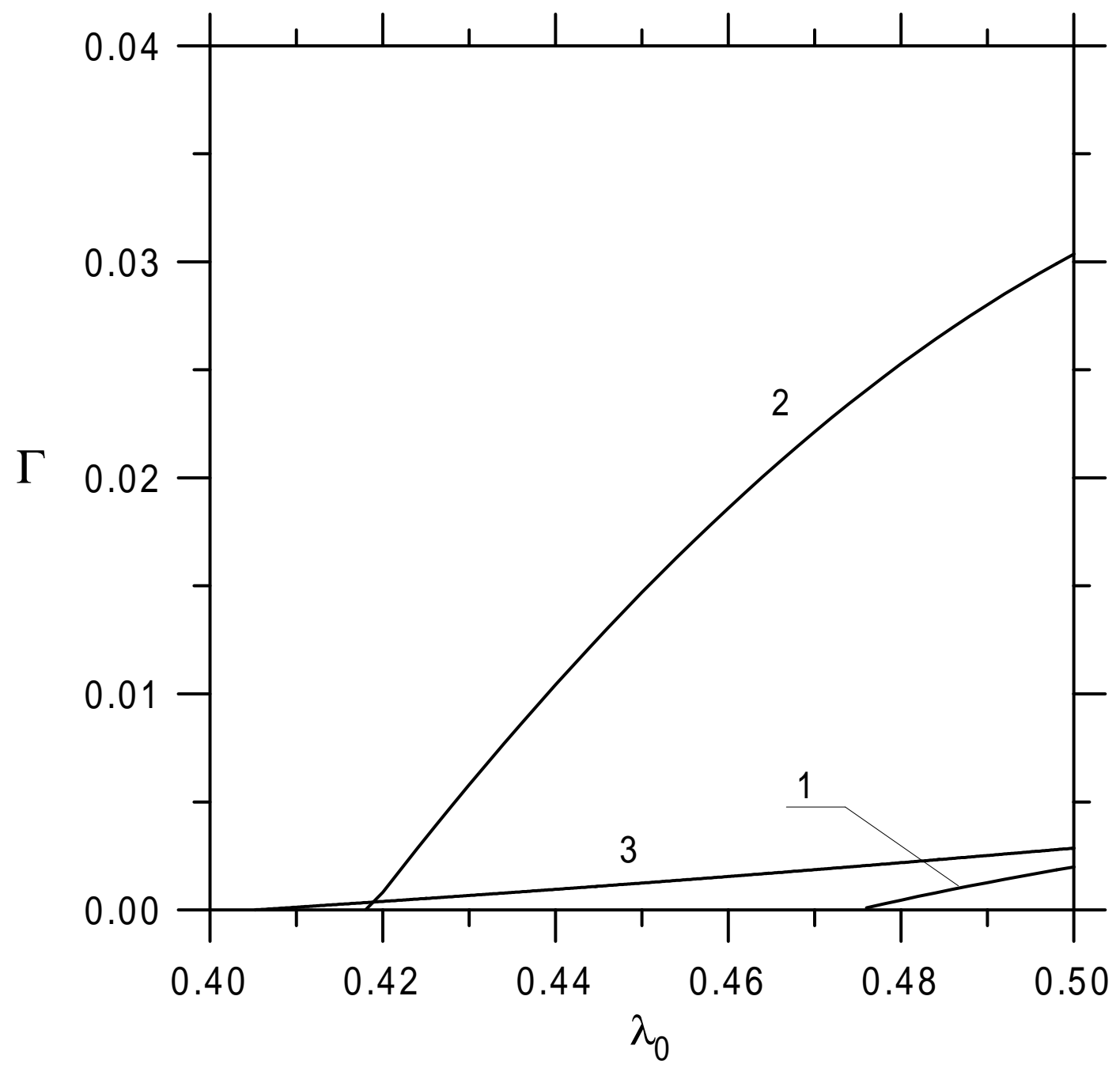

FIG. 3. The same as Fig. 2 but for $k_{\|}<0$. 


\section{External Distribution}

Plasma Research Laboratory, Australian National University, Australia

Professor I.R. Jones, Flinders University, Australia

Professor João Canalle, Instituto de Fisica DEQ/IF - UERJ, Brazil

Mr. Gerson O. Ludwig, Instituto Nacional de Pesquisas, Brazil

Dr. P.H. Sakanaka, Instituto Fisica, Brazil

The Librarian, Culham Laboratory, England

Mrs. S.A. Hutchinson, JET Library, England

Professor M.N. Bussac, Ecole Polytechnique, France

Librarian, Max-Planck-Institut für Plasmaphysik, Germany

Jolan Moldvai, Reports Library, Hungarian Academy of Sciences, Central Research Institute for Physics, Hungary

Dr. P. Kaw, Institute for Plasma Research, India

Ms. P.J. Pathak, Librarian, Institute for Plasma Research, India

Ms. Clelia De Palo, Associazione EURATOM-ENEA, Italy

Dr. G. Grosso, Instituto di Fisica del Plasma, Italy

Librarian, Naka Fusion Research Establishment, JAERI, Japan

Library, Laboratory for Complex Energy Processes, Institute for Advanced Study, Kyoto University, Japan

Research Information Center, National Institute for Fusion Science, Japan

Dr. O. Mitarai, Kyushu Tokai University, Japan

Dr. Jiangang Li, Institute of Plasma Physics, Chinese Academy of Sciences, People's Republic of China

Professor Yuping Huo, School of Physical Science and Technology, People's Republic of China

Library, Academia Sinica, Institute of Plasma Physics, People's Republic of China

Librarian, Institute of Physics, Chinese Academy of Sciences, People's Republic of China

Dr. S. Mirnov, TRINITI, Troitsk, Russian Federation, Russia

Dr. V.S. Strelkov, Kurchatov Institute, Russian Federation, Russia

Professor Peter Lukac, Katedra Fyziky Plazmy MFF UK, Mlynska dolina F-2, Komenskeho Univerzita, SK-842 15 Bratislava, Slovakia

Dr. G.S. Lee, Korea Basic Science Institute, South Korea

Institute for Plasma Research, University of Maryland, USA

Librarian, Fusion Energy Division, Oak Ridge National Laboratory, USA

Librarian, Institute of Fusion Studies, University of Texas, USA

Librarian, Magnetic Fusion Program, Lawrence Livermore National Laboratory, USA

Library, General Atomics, USA

Plasma Physics Group, Fusion Energy Research Program, University of California at San Diego, USA

Plasma Physics Library, Columbia University, USA

Alkesh Punjabi, Center for Fusion Research and Training, Hampton University, USA

Dr. W.M. Stacey, Fusion Research Center, Georgia Institute of Technology, USA

Dr. John Willis, U.S. Department of Energy, Office of Fusion Energy Sciences, USA

Mr. Paul H. Wright, Indianapolis, Indiana, USA 
The Princeton Plasma Physics Laboratory is operated by Princeton University under contract with the U.S. Department of Energy.

\author{
Information Services \\ Princeton Plasma Physics Laboratory \\ P.O. Box 451 \\ Princeton, NJ 08543
}

Phone: 609-243-2750

Fax: 609-243-2751

e-mail: pppl_info@pppl.gov

Internet Address: http://www.pppl.gov 